\title{
Movilidad urbana y calidad de vida de las personas mayores en una ciudad vertical. El caso de Valparaíso, Chile
}

\author{
ALESSANDRA OLIVI \\ > Universidad de Valparaíso, Valparaíso, Chile. alessandra.olivi@uv.cl \\ GIULIETTA FADDA \\ > Universidad de Valparaíso, Valparaíso, Chile.gfadda@vtr.net \\ VANIA REYES \\ > Universidad Diego Portales, Santiago, Chile. vania.reyes@udp.cl \\ Universidad de Valparaíso \\ Facultad de Arquitectura \\ Revista Márgenes \\ Espacio Arte Sociedad \\ Movilidad urbana y calidad de vida de las personas mayores en una \\ ciudad vertical. El caso de Valparaíso, Chile \\ Diciembre 2016 Vol $13 \mathrm{~N}^{\circ} 19$ \\ Páginas 38 a 47 \\ ISSN elec. 0719-4463 \\ ISSN imp. 0718-4034 \\ Recepción: septiembre 2016 \\ Aceptación: noviembre 2016
}

\section{RESUMEN}

Chile se encuentra entre los países de América Latina que, a lo largo de la última década, han experimentado un intenso proceso de urbanización y una rápida transición demográfica hacia el envejecimiento. Con base en la observación directa de la vida cotidiana de las personas mayores, el presente artículo explora la interrelación que se establece entre el ambiente físico, el contexto social, los recursos personales y la movilidad de las personas mayores. Los hallazgos de la investigación contribuyen a una mejor comprensión de: i. la combinación de factores que influencian las oportunidades de movilidad de las personas mayores; ii. los recursos y las estrategias que los individuos ponen en marcha para superar las barreras y redefinir la relación entre los factores que limitan sus posibilidades de movilidad; iii. la relación entre movilidad, interacción social, envejecimiento activo y calidad de vida.

PALABRAS CLAVES

personas mayores, movilidad urbana, barreras, calidad de vida, Chile

Urban mobility and Quality of Life of elderly people in a steep city. The case of Valparaiso, Chile ABSTRACT

Chile is among the Latin American countries that have seen accelerated and intense demographic transitions of aging and urbanization in the last ten years. Based on empirical evidence on the daily life of older people, the paper explores the interplay between physical and social environments, personal resources, mobility, and how these interactions impact upon their quality of life. The findings have contributed to a better understanding of: $i$. the combination of the factors that influence the opportunities for mobility of older people; ii. the resources and strategies that individuals deploy to overcome barriers and redefine the relationship between the factors that limit their possibilities for mobility, and iii. the relationship between elderly mobility and social interaction, active aging and well-being.

KEYWORDS

\section{INTRODUCCIÓN}

older people, urban mobility, barrers, quality of life, Chile

Las características del contexto socio-espacial en el cual se envejece condiciona la experiencia de la propia edad y la percepción del nivel de bienestar que tienen las personas mayores (Phillipson, 2007). Laws (1997) destaca la importancia que las prácticas espaciales tienen en el proceso de construcción de la identidad etaria y de inserción social de los adultos mayores, señalando que su apropiación territorial, así como las redes sociales a las que tienen acceso, se constituyen a través de su relación con el territorio y el espacio, siendo producto de una constante dialéctica y de un proceso de negociación continuo.
Según el modelo ecológico del envejecimiento, la calidad de vida de las personas mayores está fuertemente relacionada con el ambiente en el que habitan y en el que desarrollan sus vidas cotidianas (Lawton \& Nahemow, 1973; Peace et al., 2011). La calidad de vida es definida como un concepto multidimensional en el que se compenetran elementos objetivos y subjetivos, individuales y colectivos (Glatzer \& Zapf, 1984; Arnold, 1991; Ranzijn \& Luszcz, 2000). El concepto de calidad de vida se aplica tanto a un nivel macro (societal) así como micro (individual) (Veenhoven, 2000). Estudiar la calidad de vida de una comunidad significa analizar las con- 
diciones físicas y objetivas, así como las experiencias subjetivas y las percepciones que los individuos tienen de su existencia en su hábitat o entorno. Implica evaluar cómo viven los individuos, qué expectativas de transformación tienen de aquellas condiciones y cuál es el grado de satisfacción que ellos consiguen (García-Viniegras, 2008). Aplicando el concepto al estudio de las condiciones de vida de las personas mayores, Lawton (1983; 1991) define cuatro componentes esenciales de la calidad de vida: competencias actitudinales, condiciones medioambientales objetivas, bienestar psicológico y calidad de vida percibida. No obstante, la mayoría de los estudios que ha abordado el análisis de la relación entre la calidad de vida de las personas mayores y el ambiente se ha limitado a los aspectos objetivos, especialmente relacionados con la salud y las funciones físicas (Balfour \& Kaplan, 2002; Takano et al., 2002; Rosso et al., 2011), mientras que menor atención ha sido dedicada a la evaluación de los aspectos subjetivos (Fadda et al., 2010; Parra et al., 2011; Fadda \& Olivi, 2011).

En los últimos años, se ha registrado una mayor atención hacia la relación entre la movilidad y el bienestar de las personas mayores (Metz, 2000; 2003; Banister \& Bowling, 2004; Mollenkopf et al., 2005; Davey, 2007; Siren \& Hakamies-Blomqvist, 2009). De forma general, se asume que si bien al envejecer las personas tienden a reducir su movilidad, ésta sigue siendo un factor condicionante de la calidad de vida de las personas mayores. En la literatura sobre el tema, el concepto de envejecimiento activo se encuentra estrechamente vinculado al concepto de movilidad (Schwanen \& Páez, 2010). Para los fines de nuestro estudio, entenderemos la movilidad como la habilidad de elegir dónde, cuándo y en cuáles actividades participar a fuera del domicilio (Nordbakke, 2013:166). La movilidad implica, por lo tanto, la interacción del individuo con el contexto físico y social (Miralles-Guash \& Cebollada, 2003; Hanson, 2010). Así, las dimensiones fundamentales de la movilidad de las personas mayores identificadas en precedentes estudios (Mollenkopf et al., 2005; Schwanen et al., 2008; Nordbakke \& Schwanen, 2014) se centran en: el ambiente construido, las relaciones sociales y las normas culturales que condicionan las prácticas de movilidad.

Con base en los mencionados antecedentes, el interés de nuestro estudio se centra en explorar cómo las condiciones físicas y objetivas del entorno urbano se interrelacionan con la dimensión subjetiva y perceptiva de las personas mayores, condicionando su movilidad cotidiana y su calidad de vida. La investigación ha sido realizada en Valparaíso, ciudad que, además de encontrarse entre las tres ciudades chilenas con el mayor porcentaje de personas mayores de 60 años (INE, 2014), presenta una topografía muy peculiar que condiciona fuertemente los desplazamientos de las personas.

Tras exponer el enfoque teórico y la metodología aplicada en la investigación, el artículo analiza la forma en que la estructura físicomorfológica de la ciudad se articula con las condiciones socioeconómicas y personales de las personas mayores, condicionando sus oportunidades y estrategias de movilidad. A partir del análisis de las experiencias de movilidad cotidiana de dos personas mayores, se discuten los principales resultados y se presentan las conclusiones.

\section{EL ENFOQUE DE LAS OPORTUNIDADES DE MOVILIDAD: ACCESIBILIDAD, RECURSOS Y BARRERAS}

Como se ha planteado en la introducción, la movilidad está directa e indirectamente relacionada con la calidad de vida. Tanto el pa- radigma de las nuevas movilidades (Urry, 2002) como el enfoque de la calidad de vida aportan claridad sobre las maneras en que la movilidad puede contribuir al bienestar individual de las personas (Fotel, 2006). La movilidad, además, no sólo es central en las prácticas cotidianas de las personas y en sus relaciones sociales, sino también es funcional para comprender y describir cómo se construyen estas experiencias y relaciones y en qué manera afectan a la calidad de vida de los individuos (Burnett \& Lucas, 2010).

La movilidad no se distribuye de forma equitativa entre las personas. Por el contrario, al ser un bien que permite acceder a otros bienes, la movilidad abre potencialidades que no son igualmente accesibles para todos, constituyendo un factor más de la desigualdad en nuestras ciudades (Ascher, 2005; Hernández, 2012). Las personas mayores son un grupo especialmente sensible y vulnerable a la movilidad (Mollenkopf \& Walker, 2007). Como se ha demostrado en anteriores estudios, con el aumento de la edad la movilidad tiende a reducirse. Esta disminución está relacionada con el progresivo deterioro de las condiciones físicas y de salud y puede considerarse un efecto indeseado propio del proceso de envejecimiento (Páez et al., 2007; Hjorthlaol et al., 2010). Sin embargo, en el contexto de ciudades y espacios construidos en función de una alta movilidad, tener capacidades reducidas de movilidad puede impedir la participación en la vida económica, política y social, limitando el acceso a oportunidades, servicios y redes sociales (Kenyon et al., 2002). Según Laws (1995) la movilidad puede considerarse una de las arenas -conjuntamente con otras cuales la vivienda, el mercado del trabajo, la cultura, la atención sanitaria y el espacio construido- donde la edad es producida como proceso social y expresión de desigualdad.

Si bien se reconoce que la edad tiene efectos específicos sobre la movilidad, las oportunidades de movilidad de las personas mayores no son homogéneas, dependiendo de la combinación de múltiples factores. Según Nordbakke (2013), las oportunidades de movilidad son definidas por el conjunto de las condiciones ambientales que caracterizan a un área compleja, de las circunstancias subjetivas y de los recursos individuales de las personas. Estas oportunidades delimitan las posibilidades de participar de la vida urbana, accediendo a las actividades que ofrece la ciudad. Sin embargo, la estructura de las oportunidades de movilidad no es fija. Implícita en su definición está la posibilidad del sujeto de intervenir activamente para aumentar el alcance de sus oportunidades, desarrollando estrategias individuales o colectivas de movilidad, para satisfacer sus necesidades y deseos.

El concepto de accesibilidad resulta fundamental para comprender cómo se estructuran las oportunidades de movilidad de las personas mayores y sus prácticas espaciales (Doi et al., 2008). La accesibilidad puede ser interpretada como una práctica individual referente a la facilidad con que cada persona puede superar la distancia entre dos lugares y al número de opciones que tiene para acceder a lugares y actividades (Miralles-Guasch \& Cebollada, 2003; Jirón et al., 2010). La accesibilidad conjuga deseos y necesidades de desplazamiento (o requerimientos de movilidad) y capacidades de satisfacerlos (Gutiérrez, 2009:2).

Acorde con este marco conceptual, los factores físicos espaciales no son suficientes para enfrentar la discusión sobre cómo la movilidad incide en la calidad de vida de las personas mayores. Sus oportunidades de movilidad son definidas por la combinación de los recursos individuales y de las facilidades y limitaciones que 
presenta el contexto urbano. Así, junto con las características físico-morfológicas del espacio urbano, las habilidades físicas y las condiciones socioculturales individuales y grupales tienen un fuerte impacto sobre las formas diferenciadas en que las personas mayores plasman su movilidad. Además de las habilidades físicas individuales, especialmente relacionadas con las capacidades motrices, los factores que tienen mayor incidencia sobre la movilidad/ accesibilidad de las personas mayores están relacionados, por un lado, con aspectos socioeconómicos y culturales como el ingreso, el sector de residencia, el género, el capital social y, por otro, con la infraestructura urbana como el equipamiento, las condiciones de la viabilidad, la seguridad, el transporte público y privado (Jouffe \& Lazo, 2010; Lange Valdés, 2011; Rosso et al., 2011).

Así, las estrategias de movilidad de las personas mayores son el resultado de la negociación entre los recursos individuales y las condiciones del contexto en las que desarrollan sus prácticas espaciales. Esta relación puede aumentar o disminuir las oportunidades de movilidad, potenciando los recursos individuales o bien generando barreras que limitan la accesibilidad de los individuos a espacios, actividades y personas (Cass et al., 2005; Jirón \& ManciIla, 2013; Nordbakke, 2013).

Finalmente, los recursos individuales y las barreras contemplados en la presente investigación hacen referencia a: las habilidades físicas y las condiciones de salud (facilidad para cubrir distancias caminando); los conocimientos y competencias (poseer un permiso de conducir, conocimiento del sistema del transporte público, manejo de la tecnología); los recursos económicos (ingreso, disponibilidad económica para costear los medios de transporte y pagar la entrada a eventos o actividades); los recursos sociales y organizacionales (contar con el apoyo de familiares y amigos para desplazarse, compaginación de tareas y actividades habituales tales como compras, atención médica, cobro de la jubilación, pago de cuentas, visitas a familiares y amigos, asistencia a clubes y asociaciones); la dimensión temporal (disponibilidad de tiempo, horario de los desplazamientos diurnos y nocturnos, condiciones meteorológicas, necesidad de respetar fechas y horarios precisos, como citas médicas, cobro de jubilación, etc.). Por otro lado, entre las condiciones del medio urbano relevantes para la movilidad de las personas mayores se consideran las condiciones físico-morfológicas de la ciudad y el sector de residencia, la infraestructura y el equipamiento urbano (calles, escaleras, ascensores, aceras, paradas de buses, taxis y colectivos, espacios públicos, tráfico urbano, contaminación), el sistema de transporte y la seguridad.

\section{DATOS Y MÉTODO}

Para abordar la complejidad de las relaciones que se establecen entre el entorno físico, la movilidad y la calidad de vida de las personas mayores, se ha recurrido a la opción metodológica de la triangulación, combinando diversas fuente de información (espacio físico y personas) y técnica de recolección de datos.

Para la caracterización de las condiciones físico-morfológicas y socio-económicas de los barrios estudiados se han elaborado fichas temáticas, basadas en la observación en terreno, y mapas SIG que georeferencian los datos demográfico-territoriales y socioeconómicos de la comuna de Valparaíso, con especial referencia a la población de adultos mayores.

Para evidenciar los distintos tipos de experiencias y estrategias relacionadas con la movilidad de las personas mayores y comprender su impacto sobre la calidad de vida del grupo más anciano de la población de Valparaíso, se ha recurrido fundamentalmente a instrumentos de tipo cualitativo. Los casos de estudio y la selección de los informantes han sido definidos en función de los siguientes criterios: densidad de adultos mayores por estrato urbano, sector de residencia (plan/cerro); condición socio-económica (vulnerable/ no vulnerable); género; tercera y cuarta edad (>60 y >75). Así, por la combinación de estos criterios se han realizado 4 focus groups y un total de 27 entrevistas en profundidad a adultos mayores residentes en los 13 barrios seleccionados por el estudio. Parte de las entrevistas han sido realizadas empleando la técnica de la etnografía móvil, acompañando a los informantes en sus desplazamientos por la ciudad o en sus momentos recreativos en espacios públicos.

Los datos han sido procesados mediante la técnica de análisis de contenido. Tras identificar y codificar los principales tópicos presentes en las narraciones de los sujetos consultados, se han reconstruido los principales significados asociados a las distintas formas de movimiento e inmovilidad y su relación con el bienestar de las personas mayores.

\section{CARACTERÍSTICAS FÍSICO-MORFOLÓGICAS DE VALPARAÍSO}

Valparaíso, caso de estudio de esta investigación, es una ciudadpuerto metropolitana, con condiciones topográficas muy abruptas. Su trama urbana se dispone sobre una estrecha planicie costera, denominada "plan", rodeado por un anfiteatro conformado por cuarenta y dos cerros, abierto al Océano Pacífico. El abanico de cerros se conecta al mar por un sistema de rutas y está surcado por un régimen de quebradas convergentes al plan (Álvarez, 2001; Pino, 2012). Este hecho contribuye a dar a los cerros la condición de unidades independientes. El plan concentra el $80 \%$ de las actividades productoras de bienes y servicios y apenas el $5 \%$ de la población residente. El restante 95\% vive en los cerros. La conformación anfiteátrica hace que su centro comercial y financiero se disponga a lo largo de un eje longitudinal, al que caen en forma repartida, proporcional y regular todas las vías de los cerros. Así Valparaíso, con sus desniveles, barreras y obstrucciones, representa un espacio urbano de especial complejidad para la movilidad, siendo al mismo tiempo dotado de un gran atractivo por sus características paisajísticas.

En relación con el transporte público, como parte del soporte físico de la movilidad de sus habitantes, además de los medios motorizados -buses, taxis colectivos y taxis-, Valparaíso cuenta con un sistema propio de ascensores-funiculares que ha conferido a la ciudad una conectividad vertical que la caracteriza. Aprovechando la abrupta pendiente de los acantilados, los ascensores conectan directamente el plan con los paseos y miradores que se ubican aproximadamente en la cota $+50 \mathrm{~m}$ de los cerros. Pese a tener un recorrido muy breve pueden llegar a cubrir una gradiente de $63,5^{\circ}$. Esto permite evitar las fuertes pendientes y las sinuosas calles que suben a los cerros, facilitando y acortando los tiempos de desplazamiento peatonal.

La ciudad también cuenta, desde 1953, con un sistema de trolleybus que mantiene conectado longitudinalmente el plan de la ciudad. Además, como parte de las inversiones de transporte público interurbano, desde 2005 está activo el metro-tren de Valparaíso, que conecta la ciudad con su área metropolitana.

La escasez de espacio en la planicie costera ha favorecido históricamente la expansión urbana vertical, manifestando, como tendencia 


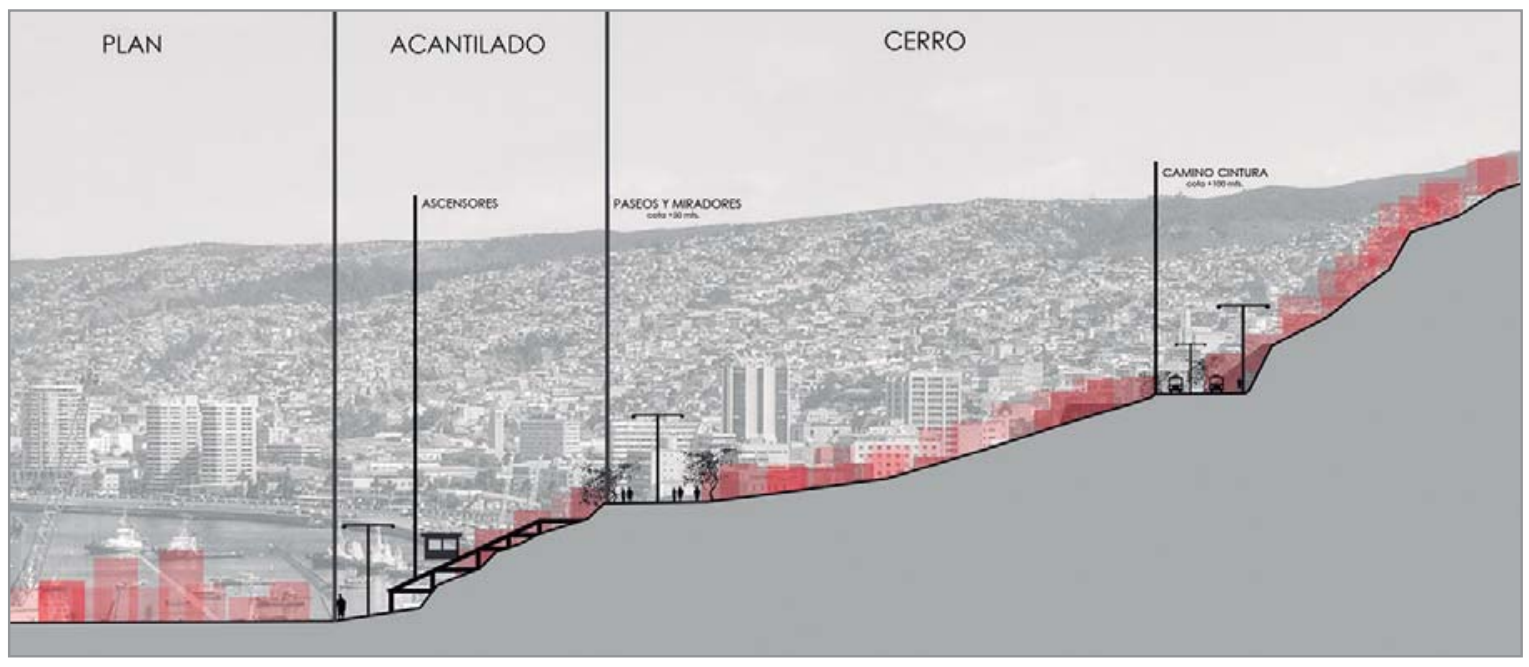

general, una marcada correlación entre el patrón de poblamiento de los cerros y la estructura de la estratificación social. Las clases sociales más acomodadas residen en la parte baja de los cerros, próxima a los paseos y miradores, mientras que los sectores más pobres se desplazan hacia la cumbre, arriba de la cota $+100 \mathrm{~m}$ y aproximadamente hasta la cota $+300 \mathrm{~m}$ (Figura 1 ). Son estos sectores los más inaccesibles y depreciados de la ciudad y es aquí donde se ubican las manzanas con mayor número de adultos mayores.

La peculiar estructura morfológica y funcional de la ciudad condiciona marcadamente la movilidad urbana cotidiana de las personas, gravando especialmente sobre las personas con movilidad reducida. La escisión del casco urbano de Valparaíso entre plan y cerros y la concentración de las centralidades -servicios básicos y financieros, comercios, mercados, espacios públicos y de diversión- en el plan imprime en los patrones generales de movilidad una direccionalidad muy definida. Mientras que las personas que residen en el plan pueden prescindir de subir a los cerros, las personas que viven en los cerros tienen que bajar al plan con una frecuencia significativa para satisfacer sus distintas necesidades. Así, de forma general, los desplazamientos ordinarios al interior de la ciudad se articulan de acuerdo a las trayectorias.

Las personas mayores consultadas hacen constante referencia a las dimensiones del cerro y del plan como a dos instancias claramente diferenciadas a través de fronteras y bordes física y socialmente definidos. En la representación de esta división se superponen elementos topográficos y administrativos, referencias físicas, sociales y simbólicas. El plan/abajo y el cerro/arriba son las dos dimensiones antagónicas y al mismo tiempo complementarias que se transforman en coordenadas utilizadas para ubicar en el tejido urbano locus habitacionales, servicios y personas y representar la pertenencia de los individuos a determinados espacios y comunidades. En Valparaíso se puede vivir abajo o arriba, más abajo o más arriba dependiendo del posicionamiento de la persona en la trama urbana.

Vivir arriba o abajo implica determinadas representaciones de nosotros y de los otros, de las redes sociales, de los espacios a los que se tiene acceso y de las efectivas posibilidades de alcanzarlos. El sector de residencia condiciona las conductas individuales de las personas mayores incidiendo diferencialmente en las estrategias de movilidad empleadas para acceder a espacios y personas. Mientras que la movilidad de las personas mayores que residen en 
el plan se despliega en sentido longitudinal, vivir en el cerro implica una movilidad vertical, caracterizada por bajadas y subidas, cuyas pendientes tienden a aumentar a medida que aumenta la distancia desde el plan. Debido a la menor habilidad motora asociada a la condición etaria, los adultos mayores resultan ser un grupo particularmente vulnerable a la influencia de las dificultades propias de las condiciones físico-morfológicas de los cerros. Las limitaciones físicas-espaciales y etarias pueden, además, superponerse a otros tipos de limitaciones, condicionando las decisiones relativas a los desplazamientos y a las estrategias de movilidad adoptadas para sortear dichas dificultades.

\section{ANÁLISIS Y DISCUSIÓN}

A partir de la reconstrucción de las experiencias habituales de movilidad de las personas mayores, a continuación se presentan dos casos, seleccionados sobre la base de las variables del estudio, que permiten ahondar en los múltiples factores y condicionamientos que delimitan las oportunidades de movilidad de estas personas, influenciando las decisiones relativas a sus desplazamientos habituales. Las estrategias de movilidad que estos sujetos orquestan para satisfacer sus necesidades básicas y recreativas son el producto de la negociación entre sus recursos individuales y las limitaciones a las que deben enfrentarse cotidianamente en sus prácticas espaciales.

\section{Caso1}

Francisco tiene 80 años y vive en el barrio El Almendral, situado en el plan de Valparaíso. Fue acogido como exiliado político en Canadá en 1973, donde trabajó como agente de aduana. Regresó a Chile en 1992, tras cumplir con los requisitos de jubilación del gobierno de Canadá. Sus ingresos, superiores a la media de los jubilados chilenos, le permiten cubrir holgadamente todas sus necesidades básicas. Separado, vive en régimen de pensión en una casa regentada por la propietaria, lo que le libera de tener que ocuparse de las tareas domésticas.

Francisco ha optado por vivir en el plan por las mejores condiciones de accesibilidad.

He preferido el plan por el fácil acceso, porque tengo acceso a todo. Yo sufro de asma, entonces el subir o bajar escaleras, como sucede en Valparaíso, no es lo mejor para vivir. Este sector es agradable. El hecho de no depender ni de ascensores ni de taxis, radio taxis, colectivos, o buses para llegar, sino que tengo simplemente todas las facilidades que toma vivir en el plan. Además, tengo el tren a 50 metros a pie de la casa y al frente de la universidad están todos los buses que te llevan a cualquier lugar. Entonces, puedo estar hasta muy tarde en otro lugar y no tengo que depender de tomar un taxi, o cualquier cosa, al vivir cerca.

Pese a ello, Francisco pasa la mayoría del tiempo en su casa, debido a que se siente muy inseguro y vulnerable al transitar por la ciudad.

Hay un temor muy grande. Yo siento latente ese temor. Andan muchos 'lanzas' [ladrones, ndt.]. Hay gente que viene a pasear acá y aparece uno por ahí y le roban las cosas, las cámaras, los bolsos. Yo no podría soportar un empujón, que me tiren al suelo [...] no voy a tener la agilidad de levantarme $y$, seguramente, los huesos viejos van a tener menor resistencia que los de un joven. Tampoco voy a salir corriendo detrás de un ladrón, porque ni siquiera voy a poder gritar. Hay una amenaza latente, hay un peligro latente.
Las amenazas asociadas a la criminalidad se suman a las que derivan de la alta densidad del tránsito vehicular en el sector donde reside y del escaso equipamiento, aumentando la percepción del riesgo asociado al transitar por las calles.

¡Aquí simplemente a uno lo atropellan! Los vehículos, los atravesamientos, los accesos a las calles, las veredas, no hay una ventaja para las personas. Y volvemos nuevamente a la tercera edad. Obviamente, no hay agilidad para correr y saltar lo que viene, o ver el vehículo que se acerca con la misma visualización de una persona más joven, por ejemplo, y eso hace que no sea tan agradable transitar en ese sentido. Porque no hay como cruzar la calle por aquí donde vivo. Hay que dar una vuelta enorme o sortear los vehículos, como lo hacen todos.

Esta sensación de inseguridad y vulnerabilidad hace que Francisco limite sus desplazamientos habituales a determinados espacios urbanos y solamente en ciertos horarios. Con frecuencia semanal acude, en horario de mañana, a la Biblioteca Municipal, recorriendo un trayecto de aproximadamente 15 cuadras. Como una forma de obligarse a hacer más ejercicio, un par de veces por semana, cuando hace buen tiempo, almuerza en un restaurante del centro, donde conoce el dueño y se queda un rato a conversar con él antes de regresar a su casa. Se desplaza siempre caminando, deteniéndose muy poco a lo largo del camino y utilizando preferentemente un paseo peatonal, por ser abierto, bien acondicionado y sombreado por palmeras. Saltuariamente acude al banco, ubicado a dos cuadras de su casa, y cada dos meses visita a su médico especialista, ubicado a 20 cuadras de su casa, siempre desplazándose a pie. Su rutina diaria se desarrolla enteramente en la casa, donde permanece cerrado como en un fuerte contra los indios en las películas del oeste, así lo visualizo. No mantiene ninguna relación con los vecinos más allá del saludo. Después de cenar, si hace buen tiempo, sale a pasear un rato sin alejarse de su cuadra.

Desde su habitación se mantiene en contacto telefónico con sus familiares y a través de internet con los amigos. Ocupa la mayoría del tiempo leyendo, escuchando música y navegando en internet. Esta última actividad le permite solventar la mayoría de sus necesidades recreativas (libros, música, películas) sin tener que desplazarse de la casa. Recibe con cierta frecuencia la visita de amigos y, en menor medida, de familiares. De vez en cuando, una amiga viene y me pasea en automóvil. Me lleva para Viña o algún otro lugar, porque me gusta tomar fotos, entonces las saco desde el auto hacia fuera o caminando muy cerca del auto. Esta es mi mayor salida.

El caso de Francisco evidencia que pese a disponer de recursos individuales suficientes para garantizarle una movilidad autónoma y satisfactoria, la sensación de inseguridad que experimenta fuera de su domicilio limita su participación en el espacio urbano y, con ello, las posibilidades de cultivar sus intereses y redes sociales. Esto le obliga a un control meticuloso de los horarios de sus desplazamientos, circunscribiéndolos a los trayectos mejor acondicionados y más seguros. Este caso confirma una tendencia registrada en otros estudios, donde el miedo del crimen (Smith et al., 2004), la inseguridad física asociada a las barreras de la infraestructura urbana (Rantakokko et al., 2010) y al caos sensorial que genera el ruido y la densidad del tráfico urbano (Parra et al., 2010) aumentan la percepción de vulnerabilidad asociada a la menor habilidad física propia de la condición etaria. El miedo generado por la combinación de estos factores puede desembocar en actitudes 


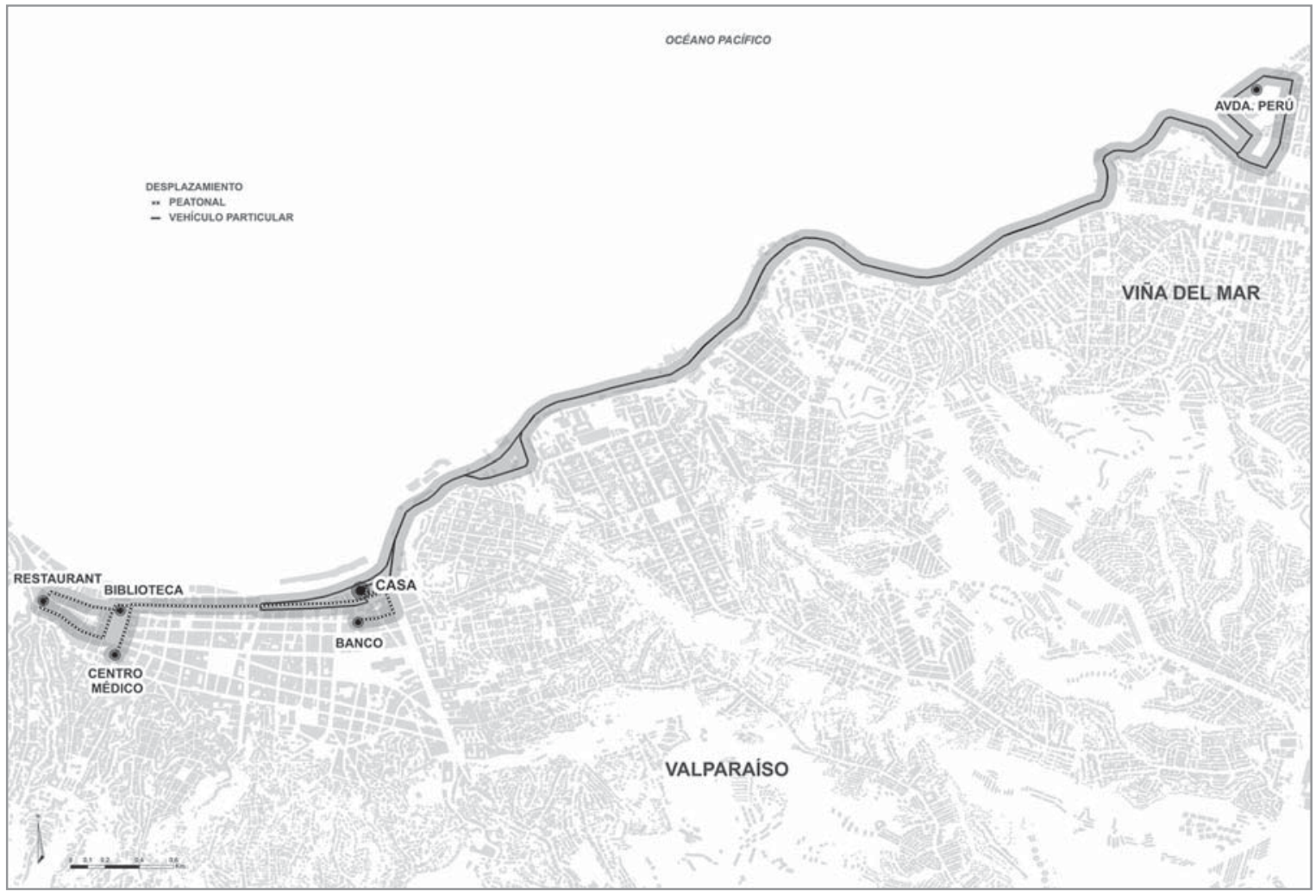

de auto-segregación. El caso de Francisco, sin embargo, subraya la importancia que tiene la activación de los recursos individuales para sortear las barreras y redefinir las oportunidades de movilidad. Además de la posibilidad esporádica de contar con amigos provistos de vehículo que lo acompañan en sus desplazamientos, permitiéndole cultivar sus intereses recreativos, es sobre todo el manejo de la tecnología lo que le permite mantener un contacto virtual pero constante con sus redes sociales y con el medio externo, paliando parcialmente su aislamiento físico.

\section{Caso 2}

Olga tiene 85 años, es viuda y vive en la parte alta del Cerro Merced donde comparte casa con su hijo y las familias de sus dos nietos. Tiene un ingreso mensual de $\$ 72.000$ que proviene de su pensión de viudez. Es poco, pero me doy vueltas bien para mi sola. Hay alguna gente que no tiene ni pensión, ni ninguna cosa. Hay que saber manejar la plata también! De eso tengo que tener para el gas, para toda clase de gastos, me arreglo el pelo, para no verme tan vieja [...] y otras cositas que tengo que tener para comida. Hago algunos trabajos extras, vendo completos acá en el barrio, hago cualquier cosita para que me alcance todo el mes.

Si hace buen tiempo, Olga baja al plan de la ciudad todos los días porque es que me aburro en la casa. Los horarios pueden variar, dependiendo de si simplemente sale a pasear o de si tiene que realizar algún trámite (cobrar la pensión, ir al consultorio). Pese a la considerable distancia que separa su casa del plan de la ciudad, su estado físico le permite bajar caminando y así ahorrar el dinero del transporte. No sube nadie a pie, ni baja nadie a pie, yo soy la única que baja a pie, porque la gente del cerro como ven que es barato, porque la bajada vale 200 pesos no más [...] pero a mí me gusta caminar y me ahorro la plata del transporte. El barrio es tranquilo, no hay ni gente en la calle. Del cerro, de mi casa, bajo a la plaza

$>$ Figura 2. Mapa de los desplazamientos habituales de Francisco. Fuente: Elaboración propia. 


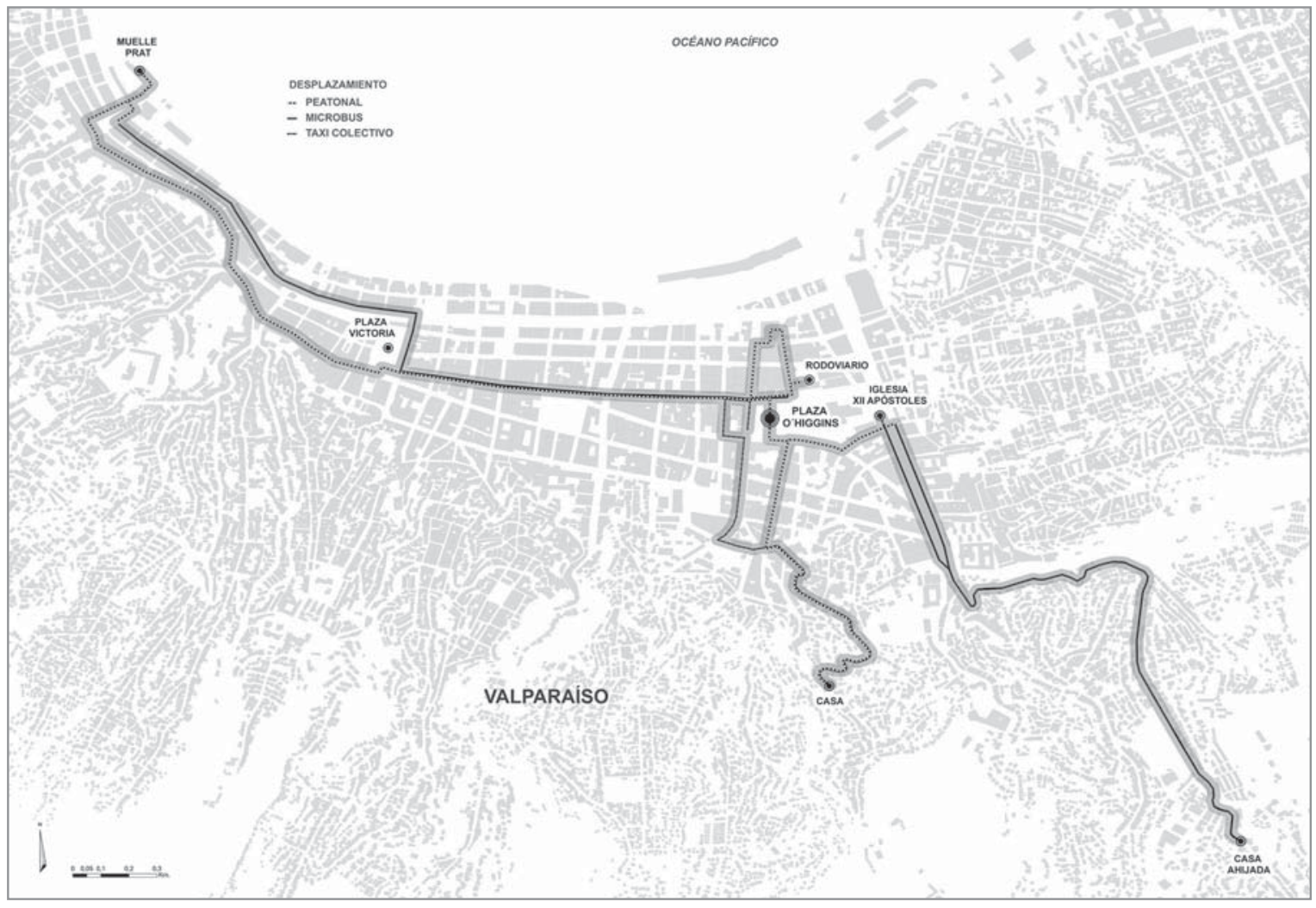

O'Higgins y acá descanso un poquito y luego depende de dónde voy a ir. Para bajar sigue la calzada de su calle y caminando llega hasta la Plaza O'Higgins, el hito principal de sus desplazamientos. Tarda aproximadamente entre media hora y 45 minutos para cubrir el recorrido de su casa hasta la plaza. Ahí se encuentra con conocidos y amigas, casi todos jubilados, con los cuales conversa, comenta noticias, comparte vivencias. En la plaza puede conseguir también unas pequeñas entradas extras, vendiendo la ración de alimentos que le entregan mensualmente en el consultorio de salud.

Desde la plaza, Olga articula sus desplazamientos por la ciudad. A veces recorre las dos cuadras hasta el mercado de abasto, para comprar algo para el pancito para cuando llego a la casa. Otras veces, se dirige hasta el Muelle Prat, sola o con una amiga, a mirar entrar los buques y salir las lanchas de turismo, porque la entrada es libre y puede ir a pasear uno [...] cuando voy al muelle, me voy en vehículo, tomamos la micro porque hay de todos los precios. Pago $\$ 210$ hasta el muelle y de vuelta me vengo caminando de allá mismo. Son como 25 cuadras, pero todas comerciales, así que una se viene entretenida mirando las vitrinas. En el trayecto de vuelta del Muelle, suele hacer una parada en la Plaza Victoria para descansar. A veces hay hombres tocando música. Yo allí no tengo ninguna amistad pero cuando regreso del Muelle me siento aquí una media horita a descansar y después sigo caminando por la avenida hasta Plaza O'Higgins otra vez. Los días que no encuentra a nadie con quien conversar en la Plaza O'Higgins, se dirige hacia el terminal de autobuses, ubicado en la cuadra del frente. Ahí me siento, unos van otros vienen, y miro a la gente: uno chico, uno grande, uno gordo y así me entretengo mirando.

Todos los domingo baja al plan para asistir a la función de las 12 en la Iglesia de los XII Apóstoles. Igual el domingo bajo un poco antes, paso a la Plaza a ver quién está, saludo, converso un poco y luego me voy a misa. Cuando termina la misma, Olga toma la micro para ir a almor-

$>$ Figura 3. Mapa de los desplazamientos habituales de Olga. Fuente: elaboración propia.
$>$ ISSN electrónico 0719-4436
$>$ ISSN impreso 0718-4034
44 > Revista Márgenes № 19 Vol 13 > Diciembre 2016: 38 a 47 Facultad de Arquitectura > Universidad de Valparaíso 
zar a la casa de su ahijada, que vive en el cerro San Roque. Yo pago \$200 no más. Le digo al chofer: - ¿Me lleva? Voy hasta la subidita no más. Porque vale \$340, pero yo pago \$200 y siempre me llevan.

Los desplazamientos de Olga no tienen una frecuencia preestablecida, ya que se van desarrollando dependiendo de las personas que encuentre en la Plaza y de la disponibilidad económica. Entonces un día voy donde mi ahijada, al otro día voy al Muelle, otro día voy al Terminal y eso para no aburrirme. Sus recorridos por la ciudad siempre comienzan y terminan en la Plaza O'Higgins, desde donde, antes de que anochezca, regresa a su casa en taxi colectivo. Me voy tardecito a la casa, ya que si es muy temprano para irme a meter a la casa me aburro. Por $\$ 250$ el colectivo me deja en la puerta. Llego allá, tomo tecito y me meto en la cama, pongo las noticias y si hay algún programa bueno lo dejo.

Olga demuestra una gran habilidad para estructurar sus estrategias de movilidad de forma independiente, activando sus recursos individuales y aprovechando las oportunidades que ofrece el contexto urbano para satisfacer sus necesidades recreativas e identitarias y mantener sus redes sociales. Sus buenas condiciones físicas le permiten superar las barreras físico-morfológicas propias del vivir en la parte alta del cerro. Asimismo, la capacidad de cubrir largas distancias caminando le permite contener el gasto de transporte, optimizando sus escasos recursos económicos. Por otro lado, el conocimiento y manejo de las redes de transporte público resulta determinante para la ampliación del radio de sus desplazamientos y la diversificación de su rutina diaria. Al aumentar las habilidades físicas y sociales y el conocimiento del sistema de transporte disminuye la percepción de vulnerabilidad asociada al espacio urbano y con ello el peso de la organización de los tiempos y la restricción de los espacio visitados. Respecto al caso anterior, Olga demuestra disfrutar de mayor libertad en sus desplazamientos y en la elección y acceso a lugares, personas y actividades. Coherentemente con los resultados de precedentes estudios (Kweon et al., 1998; Sugiyama \& Thompson, 2007; Sugiyama et al., 2009), el caso de Olga demuestra que la presencia de espacios públicos, así como los atributos estéticos y paisajísticos del espacio urbano, son factores que pueden estimular la movilidad de las personas mayores. Por las oportunidades que ofrecen en términos de interacción social, placer y disfrute estético, estos aspectos pueden repercutir positivamente sobre la dimensión social e identitaria así como sobre las condiciones físicas y de salud de las personas mayores, favoreciendo una mayor satisfacción personal y hábitos de vida más saludables.

\section{CONCLUSIONES}

Los casos de estudio ilustrados aportan a la comprensión de la combinación de factores que intervienen en la relación entre la movilidad y la calidad de vida de las personas mayores. El enfoque teórico-metodológico propuesto ha permitido ampliar la mirada físico-espacial con la cual se suele analizar la relación de las personas con el territorio que habitan, dando cuenta de cómo los aspectos objetivos y las condiciones y percepciones subjetivas condicionan la movilidad cotidiana de las personas mayores. Esto no implica desconocer la importancia de la dimensión física-espacial de la ciudad, sino analizarla a la luz de las interpretaciones y significaciones que le atribuyen los individuos que viven y se desenvuelven en ella.

En consonancia con otros estudios en este campo, nuestros hallazgos confirman la estrecha correlación entre las condiciones del contexto físico y social, los recursos individuales y las oportunidades de movilidad de las personas mayores. La relación entre estos factores tiene un fuerte impacto sobre la inserción social de los adultos mayores y la satisfacción de sus necesidades identitarias y recreativas. Su nivel de bienestar está directamente relacionado con el proceso de identificación y apropiación territorial. Las posibilidades de acceso a las distintas unidades socio-espaciales inciden sobre la percepción que los adultos mayores tienen de sí mismos y de los demás, favoreciendo un proceso de envejecimiento activo y satisfactorio en el plano personal y colectivo. Así, la desigualdad en la accesibilidad condiciona ya sea positivamente o negativamente la calidad de vida de las personas mayores.

Las evidencias empíricas producidas por el estudio muestran que entre las barreras de movilidad que enfrentan las personas mayores, las que tienen mayor peso están relacionadas con las condiciones físicas propias de la edad, tanto reales como proyectadas. Las limitaciones que derivan de la disminución de las habilidades físicas y de la percepción de una mayor fragilidad asociada al envejecimiento resultan agravadas por factores cuales la estructura morfológica y la infraestructura urbana, las condiciones de seguridad del espacio público y el sistema de transporte público. Estos aspectos adquieren especial relevancia en el caso de Valparaíso dado que la movilidad de las personas mayores depende mayoritariamente de los desplazamientos peatonales y del uso del transporte público. Pese a los beneficios para la salud que pueden derivar de una estructura urbana que induce al desplazamiento peatonal, el acondicionamiento urbano así como la calidad y el coste del transporte público resultan inadecuados para las necesidades y posibilidades de la mayoría de las personas mayores.

Los resultados del estudio indican que las restricciones de movilidad que experimentan las personas mayores no son el resultado de causas singulares, sino que son plasmadas por la combinación de distintos factores, cuya estructura no es fija ni predeterminada por las condiciones dadas, sino objeto de continuas negociaciones. En este sentido, la presencia de barreras no significa que las personas se resignen a la inmovilidad. La capacidad de los individuos de intervenir activamente para gestionar y redefinir la relación entre los factores que limitan sus posibilidades de movilidad, resulta determinante para enfrentarse a las limitaciones en el acceso a los espacios urbanos y satisfacer sus necesidades y deseos.

Finalmente, las evidencias obtenidas señalan que un mejor acondicionamiento del espacio urbano y un acceso más equitativo al sistema del transporte público son dos ámbitos de intervención que pueden mejorar las posibilidades de movilidad de las personas mayores, contribuyendo globalmente a su bienestar personal.

\section{AGRADECIMIENTOS}

Este artículo se basa en resultados de la investigación Calidad de Vida del Adulto Mayor en el Hábitat Urbano: Estudio del Caso de Valparaíso, Proyecto N 1061179, financiada por el Fondo Nacional de Ciencia y Tecnología de Chile-Fondecyt y en sus sucesivas proyecciones.

\section{BIBLIOGRAFÍA}

ÁlVAREZ, L. (2001) "Origen de los Espacios Públicos en Valparaíso: Discurso Higienista y las Condiciones Ambientales en el siglo XIX", Revista de Urbanismo 4, recuperado de http://revistaurbanismo.uchile.cl/index.php/RU/article/ viewFile/11804/12167 
ARNOLD, S. B. (1991) "Measurement of Quality of Life in the Frail Elderly", en Abeles, R. P. (ed.), The Concept and Management of Quality of Life in the Frail Elderly, pp. 50-73, NY: Academic Press.

ASCHER, F. (2005) "Ciudades con velocidad y movilidad múltiples: un desafío para los arquitectos, urbanistas y políticos", ARQ 60, pp. 11-19.

BALFOUR, J. L. \& KAPLAN, G. A. (2002) “Neighborhood environment and loss of physical function in older adults: evidence from the Alameda County Study", American Journal of Epidemiology 155(6), pp. 507-515.

BANISTER, D. \& BOWLING, A. (2004) "Quality of life for the elderly: the transport dimension”. Transport Policy 11(2), pp. 105-115.

BURNETT, P. \& LUCAS, S. (2010) "Talking, walking, riding and driving: The mobilities of older adults". Journal of Transport Geography, 18(5), pp. 596-602.

CASS, N., SHOVE, E. \& URRY, J. (2005) "Social exclusion, mobility and access", Sociological Review 53(3), pp. 539-555.

DAVEY, J. A. (2007) "Older People and Transport: Coping Without a Car", Ageing \& Society 27(1), pp. 49-65.

DOI, K., KII, M. \& NAKANISHI, H. (2008) “An integrated evaluation method of accessibility, quality of life, and social interaction", Environment and Planning B: Planning and Design 35(6), pp. 1098-1116.

FADDA, G. \& OLIVI, A. (2011) "Assessing the Quality of Life of Elderly People: Valparaiso (Chile) as a Case Study", en Heckles, J. P. \& Bergin, L. M. (eds.), Aging: Mental Aspects, Social Welfare and Health, pp. 1-47, New York: Nova Science.

FADDA, G., CORTÉS, A., OLIVI, A. \& TOVAR, M. (2010) “Perception of the values of urban space by senior citizens of Valparaiso", Journal of Aging Studies 24(4), pp. 344-357.

FOTEL, T. (2006) "Space, power and mobility: car traffic as a controversial issue in neighborhood regeneration", Environment and Planning A 38(4), pp. 733-748.

GARCÍA-VINIEGAS, C. V. (2008) Calidad de Vida. Aspectos teóricos y metodológicos, Buenos Aires: Paidós.

GLATZER, W. Y. \& ZAPF, W. (eds.) (1984) Lebensqualität in der Bundesrepublik: Objektive: Lebensbedingungen und subjektives Wohlbefinden, Darmstadt: Wissenchaftliche Buchgesellschaft.

GUTIÉRREZ, A. (2009) “Movilidad y Acceso: Embarazo y Salud Pública en la Periferia de Buenos Aires", XV Congreso Latinoamericano de Transporte Público y Urbano (CLATPU), Buenos Aires, Argentina.

HANSON, S. (2010) "Gender and mobility: new approaches for informing sustainability”, Gender, Place and Culture 17(1), pp. 5-23.

HERNÁNDEZ, D. (2012) “Activos y estructuras de oportunidades de movilidad. Una propuesta analítica para el estudio de la accesibilidad por transporte público, el bienestar y la equidad", EURE 38(115), pp. 117-135.
HJORTHOL, R., LEVIN, L. \& SIREN, A. (2010) "Mobility in different generations of older persons. The development of daily travel in different cohorts in Denmark, Norway and Sweden", Journal of Transport Geography 18(5), pp. 624-633.

INE (2014) Actualización de proyecciones de población (2002 2020), Santiago de Chile: Instituto Nacional de Estadística.

JIRÓN, P., LANGE, C. \& BERTRAND, M. (2010) "Exclusión y Desigualdad Espacial: Retrato desde la movilidad cotidiana”, Revista INVI 25(68), pp. 15-57.

JIRÓN, P. \& MANSILLA, P. (2013) “Atravesando la espesura de la ciudad: vida cotidiana y barreras de accesibilidad de los habitantes de la periferia urbana de Santiago de Chile", Revista de Geografía Norte Grande 56, pp. 53-74.

JOUFFE, Y. \& LAZO, A. (2010) "Las prácticas cotidianas frente a los dispositivos de movilidad. Aproximación política a la movilidad cotidiana de las poblaciones pobres periurbanas de Santiago de Chile", EURE 25(108), pp. 29-47.

KENYON, S., LYONS, G. \& RAFFERTY, J. (2002) "Transport and social exclusion investigating the possibility of promoting inclusion through virtual mobility", Journal of Transport Geography 10(3), pp. 207-219.

KWEON, B.-S., SULLIVAN, W. C. \& WILEY, A. R. (1998) "Green common spaces and the social integration of inner-city older adults", Environment and Behavior 30(6), pp. 832858.

LANGE VALDÉS, C. (2011) "Dimensiones culturales de la movilidad urbana”, Revista INVI 71(26), pp. 87-106.

LAWS, G. (1995) “Understanding ageism: lessons from feminism and postmodernism", The Gerontologist 35(1), pp. 112118.

LAWS, G. (1997) "Spatiality and age relations", en Jamieson, A., Harper, S. \& Victor, C. (eds.), Critical Approaches to Ageing and Later Life, pp. 90-100, Buckingham: Open University Press.

LAWTON, M. P. (1991) “A multidimensional view of quality of life in frail elders", en Birren, J., Lubben, J., Rowe, J. \& Detchman, D. (eds.), The Concept and Measurement of Quality of Life in the Frail Elderly, pp. 3-27, San Diego, CA: Academic Press.

LAWTON, M. P. (1983) "Environment and other Determinants of Well-being in Older People", The Gerontologist 23(4), pp. 349-357.

LAWTON, M. P. \& NAHEMOW, L. (1973) “Ecology and aging process", en Eisdorfer, C. \& Lawton, M. P. (eds.), The Psychology of Adult Development and Aging, pp. 619-674, Washington D. C: American Psychological Association.

METZ, D. H. (2000) "Mobility of older people and their quality of life”, Transport Policy 7(2), pp. 149-152.

METZ, D. H. (2003) "Transport policy for an ageing population", Transport Reviews 23(4), pp. 375-386.

MIRALLES-GUASCH, C. \& CEBOLLADA, Á. (2003) Movilidad y Transporte. Opciones políticas para la ciudad, Barcelona: Fundación Alternativas. 
MOLLENKOPF, H. \& WALKER, A. (eds.) (2007) Quality of Life in Old Age. International and Multi-Disciplinary Perspectives, Dordrecht: Springer.

MOLLENKOPF, H., MARCELLINI, F., RUOPPILA, I., SZÉMAN, Z. \& TACKEN, M. (eds.) (2005) Enhancing Mobility in Later Life: Personal Coping, Environmental Resources and Technical Support. The Out-of-home Mobility of Older Adults in Urban and Rural Regions in Five European Countries, Amsterdam: IOS Press.

NORDBAKKE, S. (2013) "Capabilities for mobility among urban older women: barriers, strategies and options", Journal of Transport Geography 26, pp. 166-174.

NORDBAKKE, S. \& SCHWANEN, T. (2014) “Wellbeing and mobility: a theoretical framework and literature review focusing on older people", Mobilities 9(1), pp. 104-129.

PÁEZ, A., SCOTT, D. M., POTOGLOU, D., KANAROGLOU, P. S. \& NEWBOLD, K. B. (2007) "Elderly mobility: demographic and spatial analysis of trip making in the Hamilton CMA", Urban Studies 44(1), pp. 123-146.

PARRA, D., GÓMEZ, L. F., SARMIENTO, O. L., BUCHNER, D., BROWNSON, R., SCHMID, T., GÓMEZ, V. \& LOBELO, F. (2010)“Perceived and objective neighborhood environment attributes and health related quality of life among the elderly in Bogotá, Colombia", Social Science \& Medicine 70(7), pp. 1070-1076.

PEACE, S., HOLLAND, C. \& KELLAHER, S. (2011) “'Option Recognition' in Later Life: Variations in Ageing in Place", Ageing \& Society 31, pp. 734-757.

PHILLIPSON, C. (2007) “The 'elected' and the 'excluded': sociological perspectives on the experience of place and community in old age", Ageing \& Society 27(3), pp. 321-342.

PINO, A. (2015) Quebradas de Valparaíso: Memoria social autoconstruida, Santiago: Gráfica LOM.

RANTAKOKKO, M., IWARSSON, S., KAUPPINEN, M., LEINONEN, R., HEIKKINEN, E. \& RANTANEN, T. (2010) "Quality of life and barriers in the urban outdoor environment in old age", Journal of the American Geriatrics Society 58(11), pp. 2154-2159.

RANZIJN, R. \& LUSZCZ, M. (2000) “Measurement of subjective Quality of Life of elders", International Journal of Aging and Human Development 50(4), pp. 263-278.

ROSSO, A. L., AUCHINCLOSS, A. \& MICHAEL, Y. L. (2011) "The Urban Built Environment and Mobility in Older Adults: A Comprehensive Review", Journal of Aging Research 2011 , recuperado de http://dx.doi.org/10.4061/2011/816106

SCHWANEN, T. \& PÁEZ, A. (2010) "The mobility of older people - an introduction", Journal of Transport Geography 18, pp. 591-595.

SCHWANEN, T., DIJST, M. \& KWAN, M.-P. (2008) "ICTs and the decoupling of everyday activities, space and time: introduction”, Tijdschrift voor Economische en Sociale Geografie 99 (5), pp. 519-527.
SIREN, A. \& HAKAMIES-BLOMQVIST, L. (2009) "Mobility and wellbeing in old age", Topics in Geriatric Rehabilitation 25 (1), pp. 3-11.

SMITH, A. E., SIM, J., SCHARF, T. \& PHILLIPSON, C. (2004) "Determinants of quality of life amongst older people in deprived neighborhoods", Ageing \& Society 24, pp. 793-814.

SUGIYAMA, T., THOMPSON, C. W., \& ALVES, S. (2009). “Associations between neighborhood open space attributes and quality of life for older people in Britain", Environment and Behavior 41(1), pp. 3-21.

SUGIYAMA, T. \& Thompson, C. W. (2007) "Outdoor environments, activity and the well-being of older people: conceptualising environmental support", Environment and Planning A 39(8), pp. 1943-1960.

TAKANO, T., NAKAMURA, K., \& WATANABE, M. (2002) “Urban residential environments and senior citizens' longevity in megacity areas: the importance of walkable green spaces", Journal of Epidemiology and Community Health 56(12), pp. 913-918.

VEENHOVEN, R. (2000) "Freedom and happiness: a comparative study in forty-four nations in the early 1990s", en

URRY, J. (2002) "Mobilities and proximity", Sociology 36 (2), pp. 255-274.
Movilidad urbana y calidad de vida de las personas mayores en una ciudad vertical. El caso de Valparaíso, Chile > Alessandra Olivi, Giulietta Fadda, Vania Reyes
Revista Márgenes N 19 Vol $13>$ Diclembre $2016>47$ Facultad de Arquitectura > Universidad de Valparaíso 